\title{
Spectral Reconstruction with Genetic Optimization in Case of Different Sample Sets
}

\author{
Zs. Sávoli ${ }^{1}$, A Horváth ${ }^{2}$, B. Kránicz ${ }^{1}$ \\ ${ }^{1}$ Interdisciplinary Doctoral School of Engineering, Széchenyi István University, \\ Egyetem tér 1., 9026, Győr, Hungary, e-mail: savoli@mail.barossgyor.hu
}

\section{${ }^{2}$ Department of Physics and Chemistry, Széchenyi István University, Egyetem tér 1., 9026, Győr, Hungary, e-mail: horvatha@sze.hu}

Abstract: Finding the spectral features of color sample sets is a main issue of colorimetry. It is widespread to apply Principal Component Analysis in these researches. Several studies were written about the reconstruction of samples with known spectra using principal components, and some works dealt with approximate reconstruction from tristimulus values. Our study examines how spectral reconstruction done with genetic optimization works in case of different sample sets. We have taken only the tristimulus values of the color samples given.

Keywords: spectral reconstruction, tristimulus values, principal component analysis, genetic optimization, different samle sets

\section{Introduction}

It is a basic thesis of colorimetry that any color stimulus can unequivocally be given by three numbers. These numbers can be the CIE $X, Y, Z$ tristimulus values or the coordinates of any other suitable color space $\left(Y, x, y ; L^{*}, a^{*}, b^{*} ; L^{*}, u^{*}, v^{*}\right.$ etc.). However, the description of selfluminous objects (light sources) and surfaces (secondary light sources) is often given with spectral features, in other words with spectra. This type of description provides much more information about the observed object, it needs, therefore, more than three parameters. The researchers in colorimetry started examining how they can determine or give approximately the reflection spectra of surfaces with only a few numbers. Principal Component Analysis (PCA), which is based on elements of mathematical statistics and linear algebra, has appeared to be an especially strong and interesting tool.

Several studies deal with the usage of Principal Component Analysis in colorimetry, therefore its mathematical presentation is not the subject of the present article. Publications [1]-[7] provide a general view how it works. In order to apply this method effectively, it is necessary to have a set with a large number of known spectra. Principal 
Component Analysis produces the eigenvectors belonging to the sample set. The linear combination of these eigenvectors helps to reconstruct the spectrum. The above mentioned studies present reconstruction of samples that have known and measured spectra. The question is whether it is possible to say anything about the spectrum of color samples that have unknown reflectance functions if we only know their tristimulus values $X, Y, Z$. In some of the studies focusing on this problem [8], [9], the spectral reconstruction is done with an algorithm using pseudoinvers matrix operations.

However, Principal Component Analysis or weighed Principal Component Analysis has been used in other studies. Corresponding to the tristimulus values, the three eigenvectors, whose eigenvalues are the greatest ones, are enough to get the same $X, Y, Z$ values as the result of reconstruction [10], [11]. More accurate results can be reached with more components, but they lead to undetermined equation systems, as the tristimulus values can be given in many ways from more than three components. Because of this critical statement, three vectors have been considered to be satisfactory in most cases.

Publication [12] describes our method that deals with this problem. We used the first five eigenvectors instead of the first three vectors and the features of the reflection functions of real color samples for generic optimization. It was considered that the reflection functions of the real samples are smooth, without strong oscillation and they are non-negative. This method provides a much more accurate reconstruction than the earlier ones. (Figure 1) The set of 2832 textile samples with known reflectance functions were used. Furtheremore, 148 flower samples, 565 paint samples and 8533 human skin samples were applied. Figure 2 shows these sample sets. The formalism of optimization is to be shown in brief. More details can be read in publication [12].

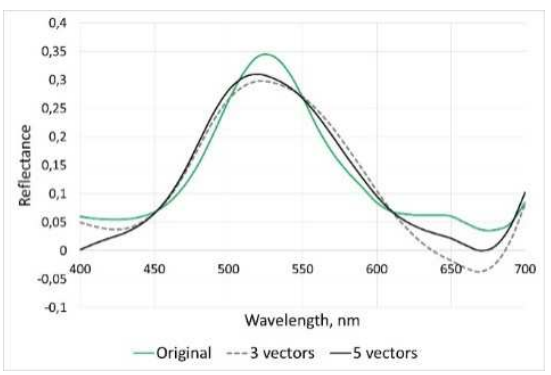

Figure 1. Reconstruction with three and with five eigenvectors

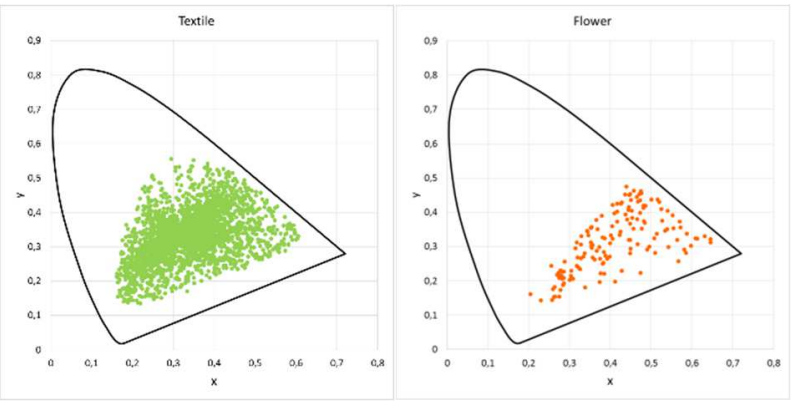




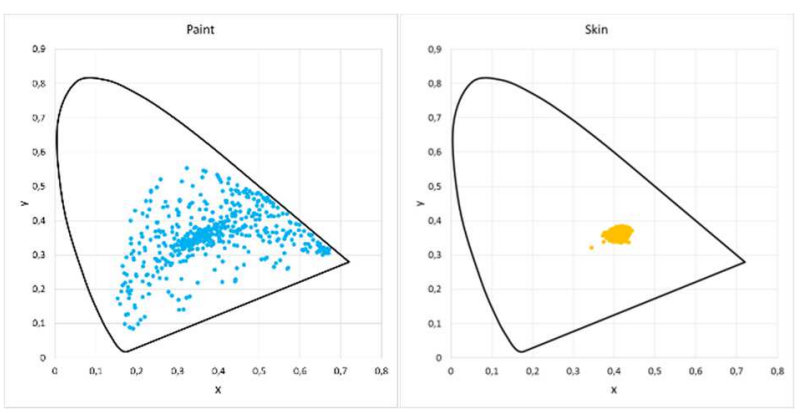

Figure 2. The textile, flower, paints, skin samples sets in CIE xy color space

\section{The formalism of optimization}

During calculation, we work with spectra (reflectance functions) whose resolution is given, therefore, we use finite-dimensional vectors instead of continuous functions. Let $N$ denote the dimension number of these vectors. For example, if we think of a spectrum with a range of $400 \mathrm{~nm}-700 \mathrm{~nm}$ and with an equidistant-wavelength step of at $10 \mathrm{~nm}, N=31$.

Let us denote the eigenvalues of the PCA method arranged in decreasing order by $\tau_{1} \geq \tau_{2} \cdots \tau_{N} \geq 0$, the eigenvectors relating to the eigenvalues by $v_{1}, v_{2}, \cdots, v_{N}$, and the mean vector by $m$. The linear combination of $M$ eigenvectors and the principal components $c_{1}, c_{2}, \cdots, c_{M}$ provides the following spectrum.

$$
f\left(c_{1}, c_{2}, \cdots, c_{M}\right)=\sum_{i=1}^{M} c_{i} \cdot v_{i}+m
$$

$f\left(c_{1}, c_{2}, \cdots, c_{M}\right)$ is also an $N$-dimensional vector.

Having $M$ fixed, variables $c_{1}, c_{2}, \cdots, c_{M}$ determine the spectrum of the reconstructed $f$ according to equation (1). As a next step, we create a function that measures the difference between this type of spectrum and the ideal spectrum. The difference is small for smooth and non-negative metamers, and it is greater and greater, if the tristimulus values deviate from the stipulated ones or if the function oscillates strongly or it takes up negative values. It is easy to calculate the tristimulus values of the spectrum by the application of color-matching functions.

$$
X_{0}=\sum_{i=1}^{N} f_{i} \cdot S_{i} \cdot \bar{x}_{i}, Y_{0}=\sum_{i=1}^{N} f_{i} \cdot S_{i} \cdot \bar{y}_{i}, Z_{0}=\sum_{i=1}^{N} f_{i} \cdot S_{i} \cdot \bar{z}_{i}
$$

In Eq. 2, $\bar{x}_{i}, \bar{y}_{i}, \bar{z}_{i}$ denote the discrete versions of the CIE color-matching functions which have the same resolution as that of the spectra, $S_{i}$.is the discrete spectral power distribution of the illuminant. Obviously, the values of $X_{0}, Y_{0}, Z_{0}$ depend on the coefficients $c_{i}$, but we do not emphasize this dependence for the sake of briefness. 
We can calculate the squared sum of the differences to show how much the values $X_{0}, Y_{0}, Z_{0}$ deviate from the predefined values $X, Y, Z$.

$$
d_{0}\left(c_{1}, c_{2}, \cdots, c_{M}\right)=\left(X-X_{0}\right)^{2}+\left(Y-Y_{0}\right)^{2}+\left(Z-Z_{0}\right)^{2}
$$

This $d_{0}$ value is non-negative and it is equal to 0 when a metamer complies with the definition. If $M=3$, the equation system of the metamer has a single solution. A lot of earlier studies which used PCA ended with giving this solution. If $M>3$, it has an infinite number of solutions, and we can choose the most realistic one by the use of constraints on negativity and strong oscillation.

We can describe the negativity of the function by the integral of the negative and the positive part, or with the ratio of their sums in the discrete case. Denote:

$$
\begin{gathered}
{f^{+}}^{+}=\max \left(f_{i}, 0\right) \\
f^{-}=\min \left(f_{i}, 0\right) \\
F^{+}=\sum_{i=1}^{N} f_{i}^{+} \\
F^{-}=-\sum_{i=1}^{N} f_{i}^{-}
\end{gathered}
$$

Definitions (4) and (5) resemble the terms of lower and upper covering functions used in analysis.

The penalty term on negativity is:

$$
P_{n}=\frac{F^{-}}{\left(F^{+}+F^{-}\right)} \cdot W_{n}
$$

$W_{n}$ is the weight factor which is used to set the relative weight of this term within the optimization function. It is obvious that $P_{n}=0$ if the function has only non-negative values and $W_{n}=1$, and the more negative parts, $f$ contains the greater positive values $P_{n}$ has got. It is 1 in extreme cases.

We define the oscillation of the function by the squared sum of the deviation between the neighboring terms:

$$
V=\sum_{i=1}^{N-1}\left(f_{i+1}-f_{i}\right)^{2}
$$

The penalty term on oscillation is 


$$
P_{v}=\sqrt{\frac{V}{(N-1)}} \cdot W_{v}
$$

The cost function whose minimum is assumed to determine the metamer with the best qualitative features is the following.

$$
d\left(c_{1}, c_{2}, \cdots, c_{M}\right)=d_{0}+P_{n}+P_{v}
$$

We can get $d_{0}$ from (3), $P_{n}$ from (8) and $P_{v}$ from (10). The weights $W_{n}$ and $W_{v}$ show the importance of one or the other penalty terms. According to the pre-calculation we have already made, the useful values are $W_{n}=100, W_{1}=1$. A little change in them will not influence the final result.

All in all, $d$ is a non-linear function with $M$ variables, whose minimum corresponds to the best function for us, in other words, vector $\left(c_{1}, c_{2}, \cdots, c_{M}\right)$ which gives the location of the extreme values, contains the optimal weight of the eigenvectors used in the reconstruction.

In order to find the minimum point of function $d$, which has been given in equation (11) above, we use our own genetic optimization program. The genetic algorithm was chosen because $d$ has a lot of local minima (mainly because of the oscillation term) and the gradient-based methods generally cannot find the global minimum in these cases.

Our genetic algorithm uses the standard genetic operators, e.g. mutation and crossing, and in order to accelerate the search for local maxima, it uses hill-climbing steps. We had already applied this code to solve more industrial optimization problems [13].

\section{Values describing the accuracy of the reconstruction}

We can describe the accuracy of the reconstruction by several measuring numbers in a quantitative way. We can measure the color difference between the examined sample and the reconstructed sample, the sepctral deviation and accuracy between the original and the reconstructed reflection funtion.

The color difference can be given as it follows. As the first step, the tristimulus values $X, Y, Z$ of the samples have to be transformed into values $L^{*}, a^{*}, b^{*}$ (12), where $X_{n}, Y_{n}, Z_{n}$ are the tristimulus values of the reference white tristimulus values under a given illuminant.

$$
\begin{gathered}
L^{*}=116 \cdot f\left(\frac{Y}{Y_{n}}\right)-16, a^{*}=500 \cdot\left(f\left(\frac{X}{X_{n}}\right)-f\left(\frac{Y}{Y_{n}}\right)\right), b^{*}=200 \cdot\left(f\left(\frac{Y}{Y_{n}}\right)-f\left(\frac{Z}{Z_{n}}\right)\right) \\
f(t)=\left\{\begin{array}{l}
t^{\frac{1}{3}}, t>\left(\frac{6}{29}\right)^{3} \\
\frac{1}{3} \cdot\left(\frac{29}{6}\right)^{2} \cdot t+\frac{4}{29}, t \leq\left(\frac{6}{29}\right)^{3}
\end{array}\right.
\end{gathered}
$$


In case of all the used samples, the condition $t>\left(\frac{6}{29}\right)^{3}$ is met. The $X_{n}, Y_{n}, Z_{n}$ values of the reference white tristimulus under the illuminant with $S(\lambda)$ spectral power distribution are given by equation (13).

$$
\begin{gathered}
X_{n}=k_{\varphi} \cdot \int_{400}^{700} S(\lambda) \cdot \bar{x}_{\omega}(\lambda) d \lambda, Y_{n}=100, Z_{n}=k_{\varphi} \cdot \int_{400}^{700} S(\lambda) \cdot \bar{z}_{\omega}(\lambda) d \lambda \\
k_{\varphi}=\frac{100}{\int_{400}^{700} S(\lambda) \cdot \bar{y}_{\omega}(\lambda) d \lambda}
\end{gathered}
$$

The normalisation coefficient is denoted by $k_{\varphi}$ and $\bar{x}_{\omega}(\lambda), \bar{y}_{\omega}(\lambda), \bar{z}_{\omega}(\lambda)$ are the CIE color matching functions. The values of the comparable color samples are $L_{1}^{*}, a_{1}^{*}, b_{1}^{*}$, and $L_{2}^{*}, a_{2}^{*}, b_{2}^{*}$. Knowing these, the CIE Lab $\Delta E_{a b}^{*}$ color difference can be determined (15).

$$
\Delta E_{a b}^{*}=\sqrt{\left(L_{2}^{*}-L_{1}^{*}\right)^{2}+\left(a_{2}^{*}-a_{1}^{*}\right)^{2}+\left(b_{2}^{*}-b_{1}^{*}\right)^{2}}
$$

If $\Delta E_{a b}^{*}=0$, the test samples are the same. If $\Delta E_{a b}^{*}=1$, it gives the just perceptible difference under a given illuminant.

Two different values are given for the spectral accuracy by the publications. One of them is a numerical value, GFC (goodness of fit coefficient) (16).

$$
G F C=\frac{\sum_{i=1}^{31} \varphi\left(\lambda_{i}\right) \cdot \varphi_{r}\left(\lambda_{i}\right)}{\sqrt{\sum_{i=1}^{31} \varphi\left(\lambda_{i}\right)^{2}} \cdot \sqrt{\sum_{i=1}^{31} \varphi_{r}\left(\lambda_{i}\right)^{2}}}
$$

$\varphi\left(\lambda_{i}\right)$ denotes the sample reflection function at wavelength $\lambda_{i} \cdot \varphi_{r}\left(\lambda_{i}\right)$ denotes the reconstructed spectrum at $\lambda_{i}$. If $G F C=1$, the reconstructed function is perfectly identical with the original one. Therefore the closer GFC gets to 1, the more accurate the spectral reconstruction is. The formula in (16) corresponds to the cosine value of the angle of two abstract vectors.

The other accuracy specifying value is $R M S$ (root mean square), which gives the reconstruction error according to the differences between the original and the reconstructed spectra (17). 


$$
R M S=\sqrt{\frac{1}{31} \cdot \sum_{i=1}^{31}\left(\varphi\left(\lambda_{\mathrm{i}}\right)-\varphi_{r}\left(\lambda_{\mathrm{i}}\right)\right)^{2}}
$$

The notations in equation (17) are the same as the notations in equation (16). The smaller the value is, the smaller the spectral error is and the greater the reconstruction accuracy is.

\section{Reconstruction in case of textile samples}

During calculation, we reconstructed some randomly-chosen textile samples with the above mentioned process and wanted to find the ideal coefficients $c_{i}$. During optimisation, we presumed the equienergetic CIE E illuminant. Figure 3 shows the reconstruction of randomly chosen samples with five eigenvectors.

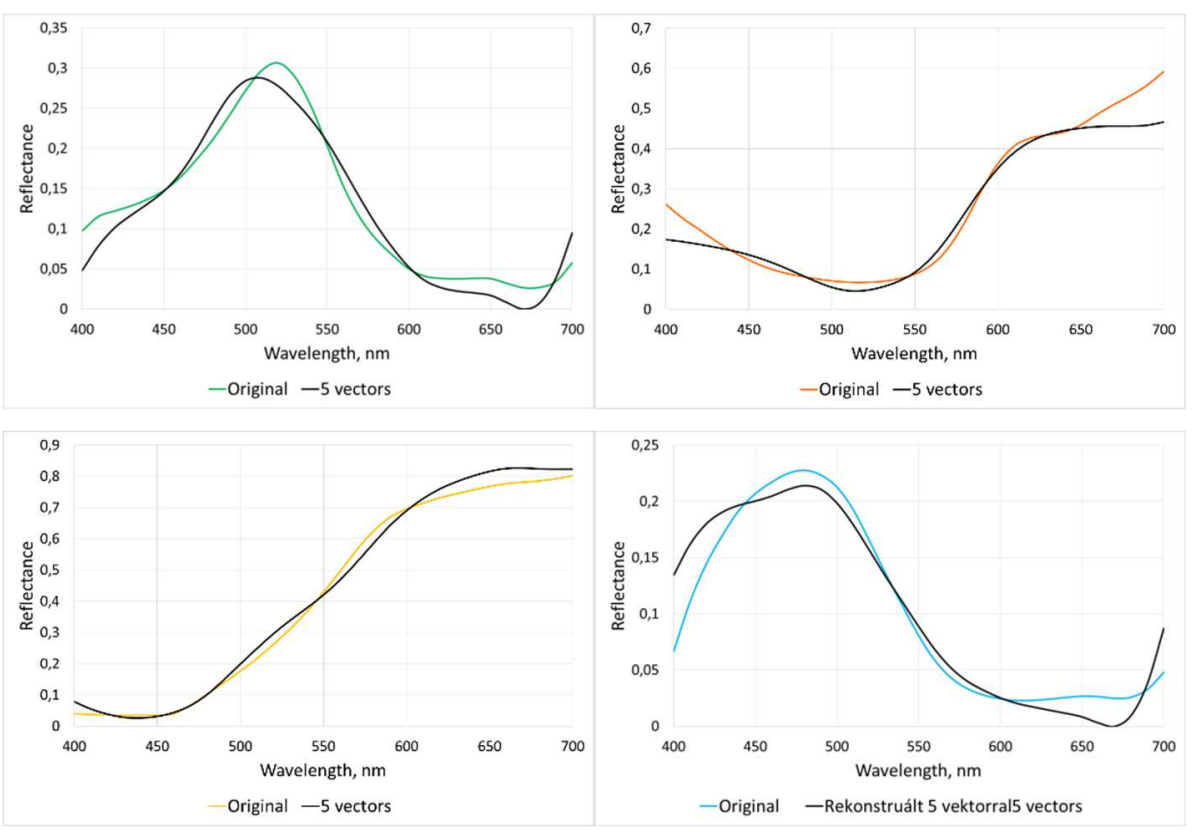

Figure 3. Reconstruction of textile samples. The black line denotes the reconstructed spectrum, the coloured line denotes the original spectrum

The following table (Table 1) shows the mean values of $\Delta E_{a b}^{*}, R M S, G F C$.

Table 1. The mean values $\Delta E_{a b}^{*}, R M S, G F C$ of the reconstruction done by genetic optimisation.

\begin{tabular}{|c|c|c|}
\hline $\boldsymbol{R M S}$ & $\boldsymbol{G F C}$ & $\boldsymbol{\Delta} \boldsymbol{E}_{\boldsymbol{a} \boldsymbol{b}}^{*}$ \\
\hline 0.0300 & 0.9926 & 0.0100 \\
\hline
\end{tabular}




\section{Reconstruction with different sample sets}

Let us examine what the coefficients and the spectra are if we use other samples than textiles. We suppose that different spectra belong to the same $X, Y, Z$ tristimulus values in cases of other materials. The whole analysis is done for three other sample sets, namely, for 148 flowers, 565 paint samples and 8533 human skin samples, and the principal components $c_{i}$ are determined with optimisation again.

However, the eigenvectors of PCA and the coefficients $c_{i}$ are different in case of different sample sets, it is interesting to know, how big the difference is between the reconstructed spectra belonging to the same tristimulus values. Therefore, the reconstruction is done for randomly-chosen tristimulus values in case of different training sample sets. The reconstruction is done for the above mentioned sample sets, the textile samples, the flowers, the paint samples and the skin samples, with their own eigenvectors using our genetic oprimisation algorythm and presuming the illuminant $C I E E$. Figure 4 shows the reconstructed spectra of the different training sample sets belonging to the same tristimulus values $X, Y, Z$.
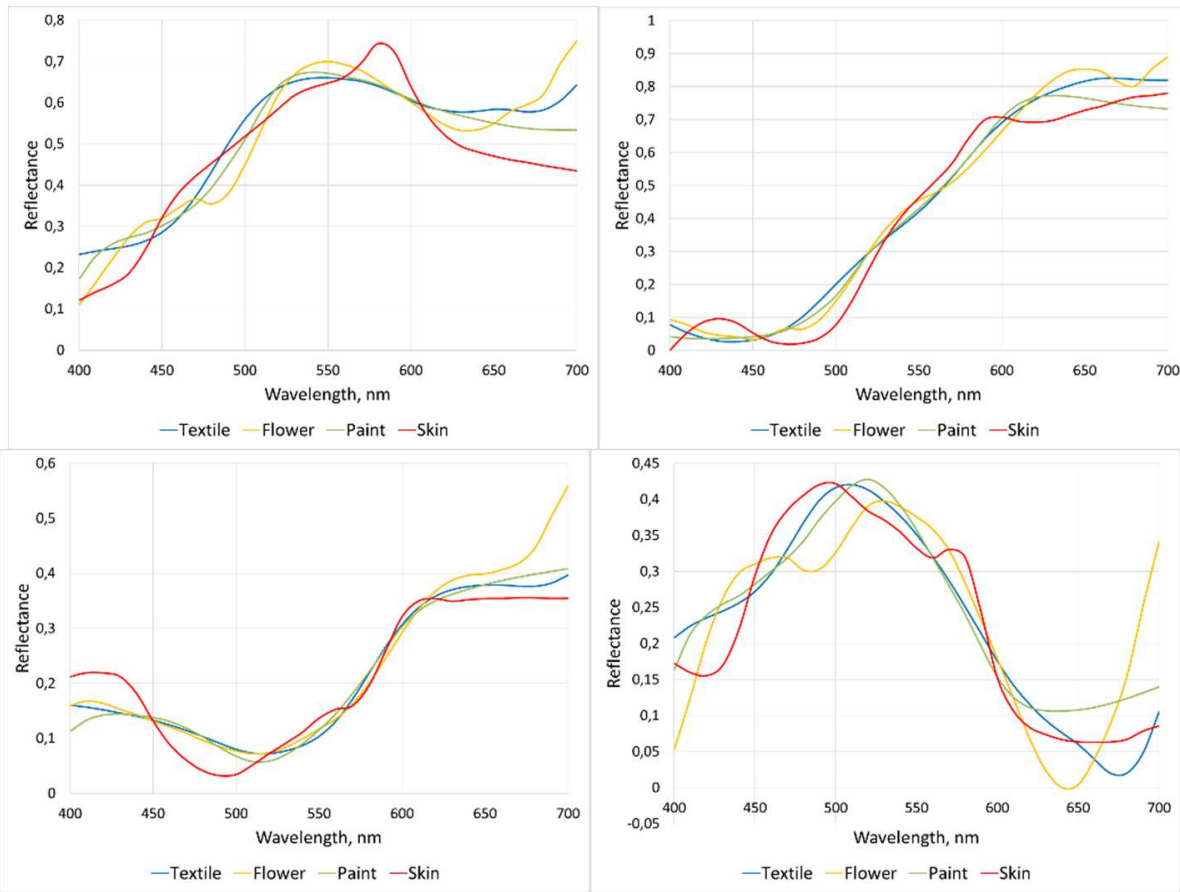

Figure 4. The reconstructed spectra belonging to given tristimulus values in case of different training sample sets

Figure 4 depicts clearly that different spectra belong to the same tristimulus values depending on the training sample sets. The reconstructed spectra are less oscillating for textile and paint samples than for flowers or human skin samples, which corresponds to the features of the samples. The reconstructed functions of the textile and paint samples resemble each other the most, however, they are not identical at all. Table 2 denotes the 
values of $R M S, G F C$ which describe the reconstruction numerically, compared to the reconstructed spectrum. With other words, the basis of comparison is always the textile spectrum. The values $\Delta E_{a b}^{*}$ are not calculated as only the shapes of the reconstructed reflectance functions are compared.

Table 2. The mean values of RMS, GFC corresponding to the same tristimulus values fro different training sets

\begin{tabular}{|c|c|c|c|c|c|}
\hline \multicolumn{2}{|c|}{ textile-flower } & \multicolumn{2}{c|}{ textile-paint } & \multicolumn{2}{c|}{ textile-skin } \\
\hline RMS & GFC & RMS & GFC & RMS & GFC \\
\hline 0.0522 & 0.9821 & 0.0325 & 0.9963 & 0.0556 & 0.9847 \\
\hline
\end{tabular}

The content of Table 2 depicts that different spectra belongs to the same tristimulus values when we use textile, flower, paint or skin samples. The differences are smaller in case of textile and paint samples than of other sample set pairs. This statement is proved for the first five eigenvectors and the mean vectors of each sample set. (Figure 5)
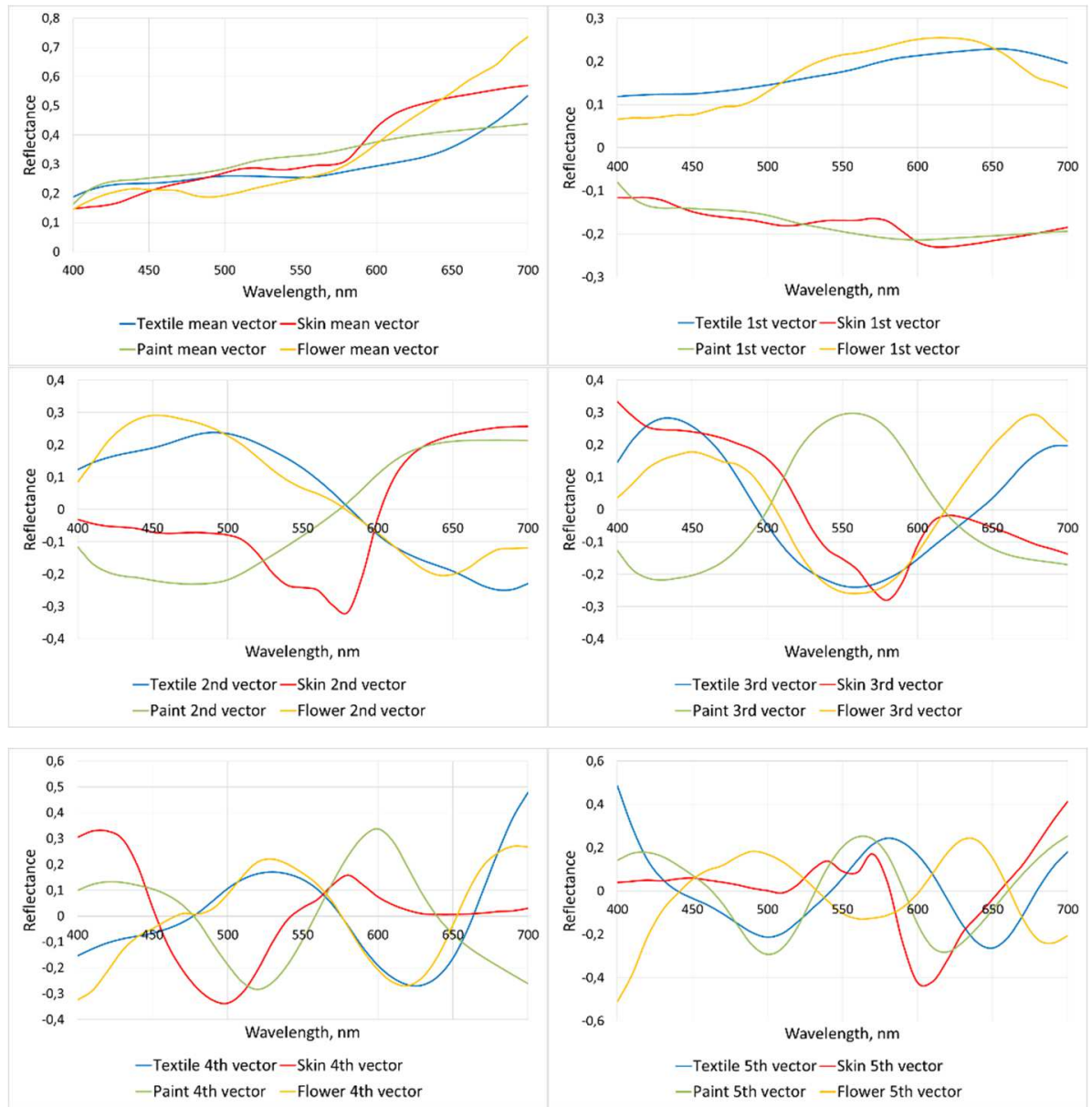

Figure 5. The mean vector and the first five eigenvectors for different sample sets 
Figure 5 depicts that the eigenvectors of the textile and the paint samples differ from each other the least, on the other hand, the eigenvectors of the other two sample sets show greater differences. Therefore, different spectra belong to the same tristimulus values $X, Y, Z$ for different sample sets.

\section{Conclusion}

Our study shows a genetic optimisation process that helps us to reconstrutct the reflectance funtion of colorful samples on the basis of their tristimulus values. During reconstruction we use the first five eigenvectors provided by the PCA together with restrictive terms for the shape of the reflectance functions of real samples. It gives the opportunity to choose those metamers from their infinite set in such a way that the reflectance function corresponds with the reflectance functions of the real samples.

All in all, our method makes it easy to reconstruct a spectrum for the tristimulus values of any colour sample with unknown spectrum. Even, we can tell what the spectrum looks like when the unknown sample is textile, flower, paint or skin. The method can be refined with the use of other sample sets.

\section{References}

[1] Tzeng DY, Berns RS: A Review of Principal Component Analysis and Its Application to Color Technology. Color Research And Application, Volume 30, Number 2, pp.84-98, 2005

[2] Maloney LT: Evaluation of linear models of surface spectral reflectance with small numbers of parameters. Journal of Optical Society of America, 3, pp. 1673-1683, 1986

[3] Jaaskelainen T, Parkkinen J, Toyooka S: Vector-subspace model for color representation. Journal of Optical Society of America, 7, pp. 725-730, 1990

[4] Vrhel MJ, Gershon R, Iwan LS: Measurement and analysis of object reflectance spectr. Color Research and Application, 19, pp.4-9, 1994

[5] García-Beltrán A, Nieves JL, Hernández-Andrés J, Romero J: Linear bases for spectral reflectance functions of acrylic paints. Color Research and Application, 23, pp.39-45, 1998

[6] Tajima J: A huge spectral characteristics database and its application to color imaging device design. Proceedings of the 6th IS\&T/SID Color Imaging Conference, IS\&T, Springfield, VA; pp. 86-89, 1998

[7] Hardeberg JY, Schmitt F, Brettel H: Multispectral color image capture using a liquid crystal tunable filter. Opt Engineering, 41, pp. 2533-2548, 2002

[8] Fairman HS, Brill MH: The principal components of reflectances. Color Research and Application, Vol. 29, No. 4, pp.104-110, 2004

[9] Harifi T, Amirshahi SH, Agahian F: Recovery of reflectance spectra from colorimetric data using principal component analysis embedded regression techniq. Opt. Rev., 15, pp. 302-308, 2008

[10] Farnaz A, Seyed AA, Seyed HA: Reconstruction of reflectance spectra using weighted principal component analysis. Color Research and Application, Vol. 33, No. 5, pp.360-371, 2008 
[11] Simone B: Reflectance spectra recovery from tristimulus valuesby adaptiveestimation with metametric shape correction. Journal of Optical Society of America, Vol. 27, No. 8, pp.1868-1877, 2010

[12] Sávoli Zs, Horváth A, Kránicz B: Színminta-halmazhoz illeszkedő, jó kvalitatív tulajdonságú metamer gyors keresése. Képfeldolgozók és Alakfelismerők Társaságának 10. országos konferenciája, Kecskemét, pp 722-737, 2015

[13] Horváth A, Horváth Z: Optimal shape design of Diesel intake ports with evolutionary algorithm. M Feistauer (szerk.) Numerical Mathematics and Advanced Applications, ENUMATH 2003 